\title{
The dynamics of prosodic adaptation between Italian conversational partners
}

Michelina Savino

Dept. of Education, Psychology, Communication, University of Bari, Italy

https://doi.org/10.36505/ExLing-2017/08/0024/000326

\begin{abstract}
This paper explores the dynamics of prosodic adaptation between speakers of Bari (BI) and Lecce (BI) varieties of Italian, in which polar question intonation contour is different as to the assignment of the rise for marking questioning: on the nuclear syllable in BI, on the phrase boundary in LI. Intonation analysis and distribution of contour types produced by BI-LI speaker pairs in a game-based interaction revealed that only BI speakers tended to accommodate their question intonation to their LI partners'. Dynamics of adaptation varied across BI speakers, hinting to different accommodation strategies which not necessarily entail overriding the rule of their native variety intonational grammar in spontaneous imitation.
\end{abstract}

Key words: Prosodic adaptation, question intonation, varieties of Italian

\section{Introduction}

Research on prosodic accommodation in verbal interaction has been mainly concentrated on quantitative measurements of acoustic-prosodic features (e.g. Levitan et al. 2015), whereas little attention has been paid to intonation contours. This paper focusses on the dynamics of prosodic adaptation by analysing the F0 contour of polar questions produced by Bari Italian (BI) and Lecce Italian (LI) speakers. This case is particularly interesting since the prototypical yes-no question tune is completely different in the two varieties as to the assignment of the functional rise for marking questioning (Savino 2012): BI has the rise on the nuclear syllable followed by either a falling or a rising terminal $\left(\mathrm{L}+\mathrm{H}^{*} \mathrm{~L}-\mathrm{L} \%\right.$ and $\mathrm{L}+\mathrm{H}^{*} \mathrm{~L}-\mathrm{H}$ $\%$, respectively), whereas LI has the rise on the phrase boundary preceded by a low or falling nuclear accent $(\mathrm{L} * \mathrm{~L}-\mathrm{H} \%$ or $\mathrm{H}+\mathrm{L} * \mathrm{~L}-\mathrm{H} \%)$. Therefore, in case of interacting BI-LI speakers pairs spontaneous imitation of the conversational partner's question contour would entail overriding the rule of the native variety intonational grammar as to the assignment of the rise for marking interrogativity. For BI speakers, an alternative strategy which would not imply overriding such a rule could consist in the selective imitation of the L-H\% boundary, thus preserving the realisation of the nuclear rise (resulting in a prevailing production of $\mathrm{L}+\mathrm{H}^{*} \mathrm{~L}-\mathrm{H} \%$ over $\mathrm{L}+\mathrm{H}^{*} \mathrm{~L}-\mathrm{L} \%$ ). Having this as a background, this study explores the dynamics of prosodic adaptation in pairs of BI and LI speakers asking polar questions during a game-based interaction.

ExLing 2017: Proceedings of 8th Tutorial and Research Workshop on Experimental Linguistics, 19-22 June, Heraklion, Crete, Greece 


\section{Method and materials}

Yes-no questions were elicited by involving pairs of BI-LI participants in the "Guess who?" game, where each participant is provided with a board having a set of pictures of fe/male characters drawn on it. The game starts with each player selecting a card from a separate pile of cards with the same characters drawn on the board, and it consists in being the first to guess which card the partner has selected, by asking exclusively yes-no questions on characters' features in order to eliminate candidates. In each recording session, participants were sitting in front of each other, wearing an AKG C520 condenser microphone headset connected to a Marantz PMD 661 digital recorder. Eye contact was inhibited, and each recording session lasted 35 min on average.

\section{Informants}

A total number of 10 speakers (5 from Bari and 5 from Lecce) participated in the recording sessions. They were all female students at the University of Bari, aged 22-24 and not familiar with each other. Gender, age and familiarity were controlled as parameters since they are been found as relevant in accommodation processes (e.g. Pardo 2006).

\section{Data annotation and measurements}

Around 1,000 polar questions (around 100 for each speaker) were collected and manually annotated by using Praat (Boersma 2001), along these levels:

- segments (utterances, nuclear words and syllables)

- tunes (nuclear pitch accents and boundary tones, AM framework).

The following measurements were performed:

- relative frequencies of contour types produced by BI and LI speakers in each pair/session (direction of adaptation);

- relative distribution of contour types in the first vs second half of the dialogue (dynamics of convergence at the overall dialogue level)

- distribution of imitated vs not imitated F0 contour in BI-LI adjacent turns where question is also textually repeated (adaptation dynamics at the turnlevel, influence of lexical imitation on tune imitation). Due to space limitations, results for this measure will not be presented here.

\section{Results}

The relative frequencies of all contours types produced by BI and LI speakers in each pair were calculated, as illustrated in Fig.1. It can be noted that, in all sessions, LI speakers show a clear tendency to not divert from using their prototypical (falling-)rising contour when asking questions, whereas BI speakers exhibit a larger variability in their question tune 
realisations. This indicates that prosodic adaptation was asymmetric, i.e. only BI speakers tended to accommodate their intonation contour to their LI conversational partners'. Only one LI speaker (LI_sp1 in Fig.1) shows an amount of rising-falling contours in her productions, but this contour type is different in tonal alignment with respect to the prototypical BI L+H*L-L\% . In fact, it is phonologically described as $\mathrm{L}^{*}+\mathrm{HL}-\mathrm{L} \%$ (i.e. with a nuclear peak later that in BI), and reported by Savino (2012) as a non-prototypical question tune in LI.

Accommodation behaviour vary across BI speakers: one speaker (BI_sp1 in Fig.1) does not show to adapt intonationally to her LI partner, since her questions are characterised exclusively by the BI nuclear $\mathrm{L}+\mathrm{H}^{*}$ followed by either an L-L\% or L-H\% boundary, with L-H\% prevailing over L-L\%. The remaining four $\mathrm{BI}$ speakers all exhibit a certain amount of the typical LI $(\mathrm{H}+)$ L*L-H\% contour: BI_sp2 and BI_sp4 both have $60 \%$ of their polar questions realised with a (fall-)rise instead of their native rise-fall or risefall-rise, whereas BI_sp5 and BI_sp3 exhibit (fall-)rises in less than $30 \%$ of the questions asked to their LI partners. As to their native BI question contour, also for these four speakers the production of $\mathrm{L}+\mathrm{H} * \mathrm{~L}-\mathrm{H} \%$ prevails over $\mathrm{L}+\mathrm{H}^{*} \mathrm{~L}-\mathrm{L} \%$. This outcome, common to all BI speakers, could be interpreted as a selective imitation of the $\mathrm{LI}(\mathrm{H}+) \mathrm{L} * \mathrm{~L}-\mathrm{H} \%$ contour.

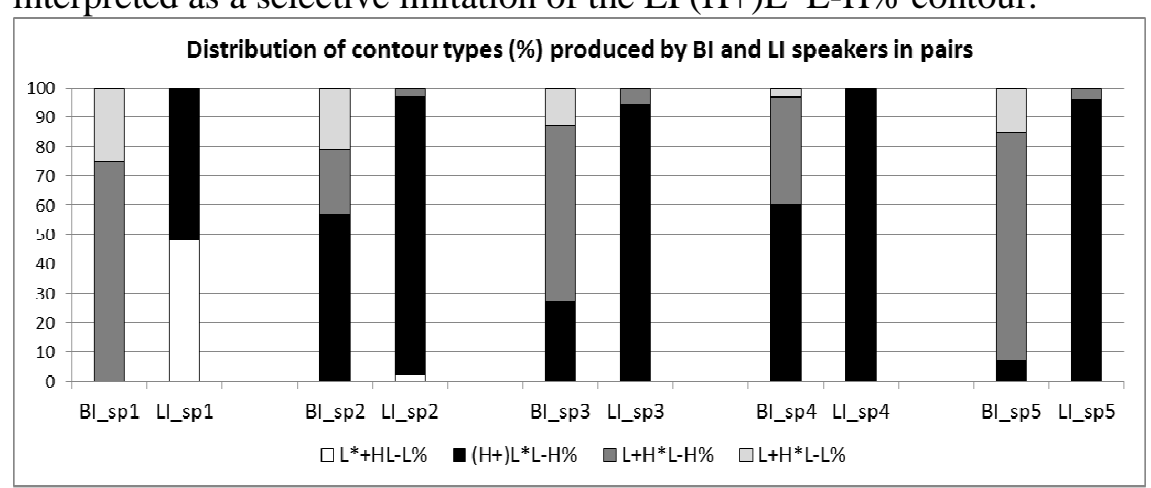

Figure 1. Distribution of question contour types (in perc.) produced by Bari Italian (BI) and Lecce Italian (LI) speakers.

Since prosodic accommodation was asymmetric, the relative frequencies of the question contour types as distributed in the first vs second half of the dialogue were determined only for BI speakers, in particular for speakers BI_sp2-3-4. Results in Figure 2 reveal two different types of accommodation dynamics across these speakers. For BI_sp3, the production of $(\mathrm{H}+) \mathrm{L} * \mathrm{~L}-\mathrm{H} \%$ contours is almost all concentrated on the second half of the game session, this implying a gradual prosodic convergence (via a underlying gradual imitation process) towards LI partner's question intonation. Instead, BI_sp3 
and BI_sp4 started realising the typical LI question contour early in their interaction, showing a rather uniform distribution of $(\mathrm{H}+) \mathrm{L} * \mathrm{~L}-\mathrm{H} \%$ between the first and the second halves of the dialogue. The latter behaviour seems to suggest for BI_sp3 and BI_sp4 a larger variability in their question intonation repertoire which could include the (fall-)rise along with the prototypical rise-fall and rise-fall-rise F0 contours: when interacting with a LI conversational partner, the (fall-)rise contour can be easily "retrieved" early in the dialogue for prosodic adaptation purposes.

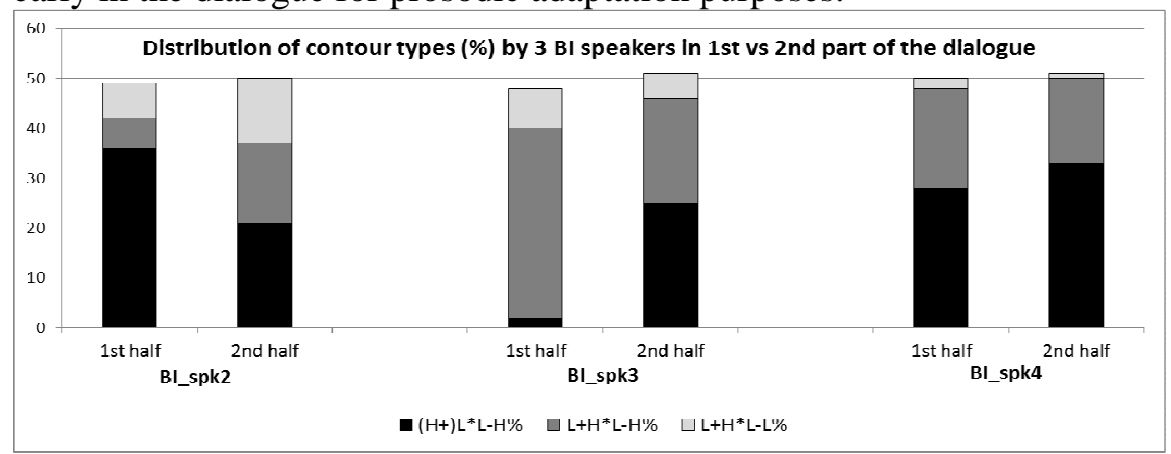

Figure 2. Distribution of question contour types (in \%) produced by BI speakers $2-4$, as occurring in the first vs the second half of the game session.

\section{Summary and conclusions}

We analysed the F0 contour of polar questions produced by BI and LI speakers pairs involved in a game-based interaction and found asymmetric adaptation, in that only BI speakers tended to accommodate their question intonation contour to their LI conversational partners'. Dynamics of prosodic adaptation can vary across BI speakers, hinting to different accommodation strategies which not necessarily entail overriding the rules of the native variety intonational grammar in spontaneous imitation of the conversational partner's question contour.

\section{References}

Boersma, P. 2001. Praat, a system for doing phonetics by computer, Glot International 5(9/10), 341-345.

Levitan, R., Benus, S., Gravano, A., Hirschberg, J. 2015. Acoustic-prosodic entrainment in Slovak, Spanish, English and Chinese: A cross-linguistic comparison. In Proc. of the SIGDIAL 2015 Conference, 325-334, Prague, Czech Rep.

Pardo, J. 2006. On the phonetic convergence during conversational interaction. JASA 119(4), 2382-2993.

Savino, M. 2012. The intonation of polar questions in Italian: Where is the rise? Journal of the International Phonetic Association 42(1), 23-48. 